\title{
A diagrammatic approach to Kronecker squares (Extended abstract)
}

\author{
Ernesto Vallejo $\|^{*}$ \\ Centro de Ciencias Matemáticas, Universidad Nacional Autónoma de México, Morelia, Mexico
}

\begin{abstract}
In this paper we improve a method of Robinson and Taulbee for computing Kronecker coefficients and show that for any partition $\bar{\nu}$ of $d$ there is a polynomial $k_{\bar{\nu}}$ with rational coefficients in variables $x_{C}$, where $C$ runs over the set of isomorphism classes of connected skew diagrams of size at most $d$, such that for all partitions $\lambda$ of $n$, the Kronecker coefficient $\mathrm{g}(\lambda, \lambda,(n-d, \bar{\nu}))$ is obtained from $k_{\bar{\nu}}\left(x_{C}\right)$ substituting each $x_{C}$ by the number of $\lambda$-removable diagrams in $C$. We present two applications. First we show that for $\rho_{k}=(k, k-1, \ldots, 2,1)$ and any partition $\bar{\nu}$ of size $d$ there is a piecewise polynomial function $s_{\bar{\nu}}$ such that $\mathrm{g}\left(\rho_{k}, \rho_{k},\left(\left|\rho_{k}\right|-d, \bar{\nu}\right)\right)=s_{\bar{\nu}}(k)$ for all $k$ and that there is an interval of the form $[c, \infty)$ in which $s_{\bar{\nu}}$ is polynomial of degree $d$ with leading coefficient the number of standard Young tableaux of shape $\bar{\nu}$. The second application is new stability property for Kronecker coefficients.

Résumé. Dans ce papier nous améliorons une méthode de Robinson-Taulbee pour calculer les coefficients de Kronecker et montrons que pour toute partition $\bar{\nu}$ de $d$ il y a un polynôme $k_{\bar{\nu}}$ avec coefficients rationels dans les variables $x_{C}$, ou $C$ est dans l'ensemble de classes d'isomorphisme des diagrammes gauches connexes de taille non plus que $d$, tel que pour toute partition $\lambda$ de $n$, le coefficient de Kronecker $\mathrm{g}(\lambda, \lambda,(n-d, \bar{\nu}))$ est obtenu de $k_{\bar{\nu}}\left(x_{C}\right)$ en substituant chaque $x_{C}$ pour le nombre de diagrammes $\lambda$-removables en $C$. Nous presentons deux applications. Premièrement nous montrons que pour $\rho_{k}=(k, k-1, \ldots, 2,1)$ et une partition $\bar{\nu}$ de taille $d$ il y a une fonction polynôme par morceaux $s_{\bar{\nu}}$ tel que pour toute $k$ on a $\mathrm{g}\left(\rho_{k}, \rho_{k},\left(\left|\rho_{k}\right|-d, \bar{\nu}\right)\right)=s_{\bar{\nu}}(k)$, et que il y a une interval de la forme $[c, \infty)$ dans lequelle $s_{\bar{\nu}}$ est polynôme de degré $d$ avec coefficient principal le nombre de tableaux de Young standard de forme $\bar{\nu}$. La seconde application est une nouveau proprieté d'estabilité des coefficients de Kronecker.
\end{abstract}

Keywords: Kronecker product, Young tableau, Schur function, Kostka number, Littlewood-Richardson rule

\section{Introduction}

Let $\chi^{\lambda}$ be the irreducible character of the symmetric group $S_{n}$ associated to a partition $\lambda$ of $n$. It is one of the major open problems in the representation theory of the symmetric group in characteristic 0 to find a combinatorial or geometric description of the multiplicity

$$
\mathrm{g}(\lambda, \mu, \nu)=\left\langle\chi^{\lambda} \otimes \chi^{\mu}, \chi^{\nu}\right\rangle
$$

\footnotetext{
*Email: valle jo@matmor. unam.mx. Supported by UNAM-DGAPA IN102611 and IN108314. 
of $\chi^{\nu}$ in the Kronecker product of $\chi^{\lambda} \otimes \chi^{\mu}$ of $\chi^{\lambda}$ and $\chi^{\mu}$ (here $\langle\cdot, \cdot\rangle$ denotes the inner product of complex characters). Seventy five years ago Murnaghan [22] published the first paper on the subject. Since then many people have searched out satisfactory ways for computing the Kronecker coefficients $\mathrm{g}(\lambda, \mu, \nu)$. However, very little is still known about the general problem.

In about the last ten years it has been discovered that Kronecker coefficients are related in an important way to two areas beyond algebraic combinatorics and representation theory. On one side there is the realization that Kronecker coefficients play an important role in geometric complexity theory [20, 21, 8]. On the other, there is the discovery that Kronecker coefficients are related to the quantum marginal problem [15, 9]. The techniques developed here could be useful in the problems on Kronecker coefficients coming from these fields.

Among the few things known, there is a method for computing arbitrary Kronecker coefficients. It was introduced by Robinson and Taulbee in [30] (see also [29, §3.4]) and reworked by Littlewood in [16]. We will refer to this method and to any of its variations as the RT method. Its main ingredients are the JacobiTrudi determinant, Frobenius reciprocity and the Littlewood-Richardson rule. Some of its applications can be found in [32, 36, 40]. Another variation of the RT method appears in [13, §6]. Some applications of this variation are given in [2, 3, 28].

The version of the RT method from [14, p. 98] suggests how to systematize it by means of the so called Littlewood-Richardson multitableaux (or simply LR multitableaux), see [10, 36]. This technique, as it is already apparent from [14], is not only useful for computations: it also lead in [37] to a combinatorial proof of a stability property for Kronecker coefficients observed by Murnaghan in [22] and to the determination of a lower bound for stability. LR multitableaux were also used in [1] to construct a one-to-one correspondence between the set 3-dimensional matrices with integer entries and given 1-marginals and the set of certain triples of tableaux.

In [36] we observed that the coefficients $\mathrm{g}(\lambda, \lambda, \nu)$ of Kronecker squares could be computed by means of a graphical approach derived from the RT method and gave explicit formulas for all partitions $\nu$ of depth $\mathrm{d}(\nu) \leq 3$. We include them in Section 7 . Some had appeared before in an algebraic but equivalent form in [12, 32, 40]; some have already been applied in [4, 5, 6, 27]; others may be suitable for future applications.

In this abstract we show that the phenomenon observed in [36] holds in full generality (Theorem 7.2). Namely, for each partition $\bar{\nu}$ of size $d$, there is a polynomial $k_{\bar{\nu}}\left(x_{C}\right)$ with rational coefficients in variables $x_{C}$, where $C$ runs over the set of isomorphism classes of connected skew diagrams of size $|C| \leq d$, such that for each partition $\lambda$ of $n$, the Kronecker coefficient $\mathrm{g}(\lambda, \lambda,(n-d, \bar{\nu}))$ is obtained from $k_{\bar{\nu}}\left(x_{C}\right)$ by evaluating each $x_{C}$ at the number $\mathrm{r}_{\lambda}(C)$ of partitions $\alpha$ of $n-d$ contained in $\lambda$ such that $\lambda / \alpha$ is isomorphic to $C$. These polynomials do not depend on $\lambda$ or $n$. In fact, we also show (Theorem 7.3 that $k_{\bar{\nu}}\left(x_{C}\right)$ can be modified to obtain another polynomial $\widetilde{k}_{\bar{\nu}}\left(t_{B}\right)$ in variables $t_{B}$, where $B$ runs over the set of isomorphism classes of connected border strips of size $|B| \leq d$. Then a similar evaluation of $\widetilde{k}_{\bar{\nu}}\left(t_{B}\right)$ also yields the corresponding Kronecker coefficient. Theorem 7.2 is derived from Theorem 7.1 which is an enhancement of the RT method described above that gives a closed combinatorial formula (up to signs) of Kronecker coefficients. It incorporates the notion of $\lambda$-removable diagram and a convenient use of special border strip tableaux. We will show its utility in Sections 8 and 9 Its ingredients are the RT method, Eg̃eciog̃lu-Remmel's combinatorial description of the entries of the inverse Kostka matrix [11], the new notion of $\lambda$-removable diagram introduced in Section 5 and Theorem 5.10. This approach should be contrasted with Murnaghan's [22, 23], where for any two partitions $\lambda=(n-a, \bar{\lambda}), \mu=(n-b, \bar{\mu})$ of 
$n$ a method is given to compute the expansion $\chi^{\lambda} \otimes \chi^{\mu}$ in terms of $\bar{\lambda}$ and $\bar{\mu}$.

Besides we present two applications of the diagrammatic approach developed here. The first is a contribution to the solution of Saxl conjecture studied for the first time in [27]. Let $\rho_{k}$ be the staircase partition $(k, k-1, \ldots, 2,1)$ and let $n_{k}$ be its size. Saxl conjecture asserts that the Kronecker product $\chi^{\rho_{k}} \otimes \chi^{\rho_{k}}$ contains every irreducible representation of $S_{n_{k}}$ as a component. Here we show what we believe to be a surprising result: Theorem 8.2, which asserts that for each partition $\bar{\nu}$ of size $d$ there is a piecewise polynomial function with rational coefficients $s_{\bar{\nu}}:[0, \infty) \longrightarrow \mathbb{R}$ such that

$$
\mathrm{g}\left(\rho_{k}, \rho_{k},\left(n_{k}-d, \bar{\nu}\right)\right)=s_{\bar{\nu}}(k)
$$

for all $k$ such that $\left(n_{k}-d, \bar{\nu}\right)$ is a partition. This is the more surprising since the product $\chi^{\rho_{k}} \otimes \chi^{\rho_{k}}$ seems to be the most difficult product of size $n_{k}$ to evaluate (see [16, p. 93]). A further analysis (Theorem 8.4 shows that $\mathrm{g}\left(\rho_{k}, \rho_{k},\left(n_{k}-d, \bar{\nu}\right)\right)$ is positive for all but at most $2 d$ values of $k$. The second result (Theorem 9.2 shows a new stability property of Kronecker coefficients that is evident once we know Theorem 7.1 The results presented in this abstract appear in full detail in the paper [39].

The abstract is organized as follows. Sections 2 and 3 contain the definitions, notation and some known results on partitions, tableaux and characters of the symmetric group used in the abstract. In Section 4 we relate LR multitableaux to Kronecker coefficients. Theorem 4.3 has not been published before, but it appears already in a similar form in [36, Corollary 4.3]. In Section 5 we introduce the notion of $\lambda$-removable diagram (Paragraph 5.4). This is the fundamental concept for our diagrammatic method. In Theorem 5.10 we show that for each isomorphism class $D$ of skew diagrams, the number $r_{\lambda}(D)$, of $\lambda$-removable diagrams in $D$, can be expressed as a polynomial with rational coefficients in variables $\mathrm{r}_{\lambda}(C)$, where $C$ runs over the set of all isomorphism classes of connected skew diagrams of size $|C| \leq|D|$. Section 6 contains a formula that expresses the number $\operatorname{Ir}(\lambda, \lambda ; \pi)$ as a polynomial with rational coefficients in the numbers $r_{\lambda}(D)$. Section 7 is the core of the paper. It contains Theorems 7.1. 7.2 and 7.3 already mentioned. We also include two formulas for $\mathrm{g}(\lambda, \lambda,(n-d, \bar{\nu}))$ when $\lambda$ is a rectangle of size $n$ and $\bar{\nu}$ is either $(d)$ or $\left(1^{d}\right)$. In Section 8 we present our contribution to Saxl conjecture, a table with the polynomials $s_{\bar{\nu}}$ for all $|\bar{\nu}| \leq 5$ and some conjectures. Finally, Section 9 contains a new stability property for Kronecker coefficients.

\section{Partitions and tableaux}

We assume the reader is familiar with the standard results in the combinatorics of Young tableaux (see for example [18, 31, 33]).

We denote by $\mathbb{N}$ the set of positive integers and for any $n \in \mathbb{N},[n]=\{1, \ldots, n\}$. If $\lambda$ is a partition, we denote its size by $|\lambda|$ and its length by $\ell(\lambda)$. If $|\lambda|=n$, we also write $\lambda \vdash n$. The depth of $\lambda$ is $\mathrm{d}(\lambda)=|\lambda|-\lambda_{1}$. For any composition $\pi=\left(\pi_{1}, \ldots, \pi_{r}\right)$ denote $\bar{\pi}=\left(\pi_{2}, \ldots, \pi_{r}\right),|\pi|=\pi_{1},+\cdots+\pi_{r}$ and $\ell(\pi)=r$. If $|\pi|=n$, we also write $\pi \vDash n$. Thus, for a partition $\lambda$ one has $\mathrm{d}(\lambda)=|\bar{\lambda}|$. Given two partitions $\lambda, \mu$ of $n$ we write $\lambda \geqslant \mu$ to indicate that $\lambda$ is greater than or equal to $\mu$ in the dominance order of partitions. The partition $\lambda^{\prime}$ conjugate to $\lambda$ is obtained by transposing the diagram of $\lambda$.

The number of semistandard Young tableaux of shape $\lambda$ and content $\pi$ is denoted by $K_{\lambda \pi}$. Let us arrange the partitions of $n$ in reverse lexicographic order and form the matrix $K_{n}=\left(K_{\lambda \mu}\right)$. It is invertible over the integers. Let $K_{n}^{-1}=\left(K_{\lambda \mu}^{(-1)}\right)$ denote its inverse. We now explain the combinatorial description of the numbers $K_{\lambda \mu}^{(-1)}$ given in [11]. Recall that a border strip is a connected skew diagram that contains 
no subset of the form $\boxplus$. A special border strip tableau $T$ of shape $\mu$ is an increasing sequence of partitions

$$
\varnothing=\mu(0) \subset \mu(1) \subset \cdots \subset \mu(c)=\mu,
$$

such that each $\mu(j) / \mu(j-1)$ is a special border strip of $\mu$, namely a border strip that intersects the first column of $\mu$. The sign of $T$ is

$$
\operatorname{sgn}(T)=\prod_{j}(-1)^{(\mathrm{ht}(\mu(j) / \mu(j-1)))},
$$

where the height ht $(\mu(j) / \mu(j-1))$ is the number of rows of $\mu(j) / \mu(j-1)$ minus one. The content, denoted by $\gamma(T)$, is the sequence

$$
|\mu(1) / \mu(0)|, \ldots,|\mu(c) / \mu(c-1)|
$$

of sizes of the special border strips arranged in decreasing order. Later it will be convenient to work with a reordering of $\gamma(T)$. Denote by SBST $(\mu)$ the set of all special border strip tableaux of shape $\mu$. Eg̃eciog̃lu and Remmel showed the following

Theorem 2.1 ([11]) For any pair of partitions $\lambda, \mu$ of $n$ one has

$$
K_{\lambda \mu}^{(-1)}=\sum_{T \in \operatorname{SBST}(\mu), \gamma(T)=\lambda} \operatorname{sgn}(T) .
$$

The number of Littlewood-Richardson tableaux (or simply LR tableaux) of shape $\lambda / \alpha$ and content $\mu$ will be denoted by $c_{\mu}^{\lambda / \alpha}$. More generally, a sequence $T=\left(T_{1}, \ldots, T_{r}\right)$ of tableaux is called a $L R$ multitableau of shape $\lambda / \alpha$ if there is a sequence of partitions

$$
\alpha=\lambda(0) \subset \lambda(1) \subset \cdots \subset \lambda(r)=\lambda,
$$

such that $T_{i}$ is LR tableau of shape $\lambda(i) / \lambda(i-1)$, for all $i \in[r]$. The content of $T$ is the sequence $(\rho(1), \ldots, \rho(r))$, where $\rho(i)$ is the content of $T_{i}$; the type of $T$ is the composition $(|\rho(1)|, \ldots,|\rho(r)|)$. The number of LR multitableaux of shape $\lambda / \alpha$ and content $(\rho(1), \ldots, \rho(r))$ will be denoted by $c_{(\rho(1), \ldots, \rho(r))}^{\lambda / \alpha}$.

\section{Characters of the symmetric group}

We assume the reader is familiar with the standard results in the representation theory of the symmetric group (see for example [14, 18, 31, 33]).

For any partition $\lambda \vdash n$, denote by $\chi^{\lambda}$ the irreducible character of $S_{n}$ associated to $\lambda$, and, for any composition $\pi=\left(\pi_{1}, \ldots, \pi_{r}\right)$ of $n$, by $\phi^{\pi}=\operatorname{Ind}_{\mathrm{S}_{\pi}}^{\mathrm{S}_{n}}\left(1_{\pi}\right)$ the permutation character associated to $\pi$. That is, $\phi^{\pi}$ is the character induced from the trivial character of the Young subgroup $\mathrm{S}_{\pi}=\mathrm{S}_{\pi_{1}} \times \cdots \times \mathrm{S}_{\pi_{r}}$ to $\mathrm{S}_{n}$. Note that if a composition $\rho$ of $n$ is a reordering of $\pi$, then $\phi^{\pi}=\phi^{\rho}$. Also, for a partition $\alpha \subseteq \lambda$, let $\chi^{\lambda / \alpha}=\sum_{\nu} c_{\nu}^{\lambda / \alpha} \chi^{\nu}$.

Definition 3.1 Let $\nu=\left(\nu_{1}, \ldots, \nu_{r}\right)$ be a partition and $T \in \operatorname{SBST}(\nu)$. Denote by $\tau(T)$ the vector $\left(t_{1}, \ldots, t_{l}\right)$ such that $t_{1}$ is the size of the border strip that contains the square $\left(1, \nu_{1}\right)$ and $t_{2}, \ldots, t_{l}$ are the sizes of the remaining special border strips arranged in non-increasing order. The vector $\tau(T)$ is not 
a partition in general, but for $\nu_{1}$ big enough it is. It will be useful to work with $\tau(T)$ as the content of $T$ instead of the usual content $\gamma(T)$ defined above, which by definition is always a partition. Let also denote $\bar{\tau}(T)=\left(t_{2}, \ldots, t_{l}\right)$ and $e(T)=|\bar{\tau}(T)|$.

Definition 3.2 Let $\bar{\nu}=\left(\nu_{2}, \ldots, \nu_{r}\right) \vdash d$. Denote $\widetilde{\nu}=\left(\nu_{2}, \nu_{2}, \ldots, \nu_{r}\right)$. Let $n \geq d+\nu_{2}$. For any $T \in \operatorname{SBST}(\widetilde{\nu})$ we define a new tableau $B_{n}(T) \in \operatorname{SBST}((n-d, \bar{\nu}))$ as follows. To the border strip in $T$ of size $t_{1}$ that contains the square $\left(1, \nu_{2}\right)$ we add the squares $\left(1, \nu_{2}+1\right), \ldots,(1, n-d)$ and form a new border strip of size $t_{1}+n-d-\nu_{2}$. Then $B_{n}(T)$ is defined as the tableau formed by this new border strip plus the remaining border strips of $T$.

Then we have

Lemma 3.3 Let $\bar{\nu}$ be a partition of $d$. Then for any $n \geq d+\nu_{2}$ we have

$$
\chi^{(n-d, \bar{\nu})}=\sum_{T \in \operatorname{SBST}(\widetilde{\nu})} \operatorname{sgn}(T) \phi^{\tau\left(B_{n}(T)\right)} .
$$

\section{Littlewood-Richardson multitableaux}

Let $\pi=\left(\pi_{1}, \ldots, \pi_{r}\right)$ be a composition of $n$. Also let $\lambda, \alpha, \mu, \beta$ be partitions such that $\alpha \subseteq \lambda, \beta \subseteq \mu$, $|\lambda / \alpha|=n$ and $|\mu / \beta|=n$. Define

$$
\operatorname{Ir}(\lambda / \alpha, \mu / \beta ; \pi)=\sum_{\rho(1) \vdash \pi_{1}, \ldots, \rho(r) \vdash \pi_{r}} c_{(\rho(1), \ldots, \rho(r))}^{\lambda / \alpha} c_{(\rho(1), \ldots, \rho(r))}^{\mu / \beta} .
$$

This is the number of pairs $(S, T)$ of LR multitableaux of shape $(\lambda / \alpha, \mu / \beta)$, same content and type $\pi$.

The following lemma is a consequence of Frobenius reciprocity and the Littlewood-Richardson rule.

Lemma 4.1 Let $\lambda, \mu, \alpha, \beta, \pi$ be as above. Then

$$
\operatorname{Ir}(\lambda / \alpha, \mu / \beta ; \pi)=\left\langle\chi^{\lambda / \alpha} \otimes \chi^{\mu / \beta}, \phi^{\pi}\right\rangle .
$$

Proposition 4.2 Let $\bar{\nu}=\left(\nu_{2}, \ldots, \nu_{r}\right)$ be a partition of $d$ and $n \geq d+\nu_{2}$. Then, for any partitions $\lambda, \mu$ of $n$ we have

$$
\mathrm{g}(\lambda, \mu,(n-d, \bar{\nu}))=\sum_{T \in \operatorname{SBST}(\widetilde{\nu})} \operatorname{sign}(T) \operatorname{lr}\left(\lambda, \mu ; \tau\left(B_{n}(T)\right)\right) .
$$

Theorem 4.3 Let $\lambda, \mu, \alpha, \beta$ be partitions such that $\alpha \subseteq \lambda, \beta \subseteq \mu$ and both $\lambda / \alpha$ and $\mu / \beta$ have size $n$. If $\rho, \sigma$ are partitions of $n$ and $\rho \leqslant \sigma$, then

$$
\operatorname{Ir}(\lambda / \alpha, \mu / \beta ; \rho) \geq \operatorname{Ir}(\lambda / \alpha, \mu / \beta ; \sigma) .
$$

\section{Diagram classes}

Definition 5.1 Let $\rho, \sigma$ be skew diagrams. Denote by $\rho=\cup_{i \in[m]} \rho_{i}, \sigma=\cup_{j \in[n]} \sigma_{j}$ their decompositions into connected components. Then $\rho$ and $\sigma$ are called isomorphic diagrams if $m=n$ and there is a permutation $\pi \in \mathrm{S}_{m}$ such that, for each $i \in[m]$, there is a bijective translation $f_{i}: \rho_{i} \longrightarrow \sigma_{\pi(i)}$. The map $f: \rho \longrightarrow \sigma$ defined, for each $x \in \rho_{i}$, by $f_{i}(x)$ is called an isomorphism of diagrams. Clearly $f^{-1}$ is also an isomorphism of diagrams. 
Definitions 5.2 Let $\lambda=\left(\lambda_{1}, \ldots, \lambda_{p}\right), \mu=\left(\mu_{1}, \ldots, \mu_{q}\right), \alpha=\left(\alpha_{1}, \ldots, \alpha_{a}\right)$ and $\beta=\left(\beta_{1}, \ldots, \beta_{b}\right)$ be partitions such that $\alpha \subseteq \lambda$ and $\beta \subseteq \mu$. If $a<p$ or $b<q$ we let $\alpha_{p+1}, \ldots, \alpha_{p}$ and $\beta_{b+1}, \cdots, \beta_{q}$ be zero. The disjoint union $\lambda / \alpha \sqcup \mu / \beta$ of $\lambda / \alpha$ and $\mu / \beta$ is the skew diagram $\nu / \gamma$ where

$$
\nu=\left(\lambda_{1}+\mu_{1}, \ldots, \lambda_{1}+\mu_{q}, \lambda_{1}, \ldots, \lambda_{p}\right) \text { and } \gamma=\left(\lambda_{1}+\beta_{1}, \ldots, \lambda_{1}+\beta_{q}, \alpha_{1}, \ldots, \alpha_{p}\right) .
$$

For example, if $\lambda=(3,2,1), \alpha=\left(1^{2}\right), \mu=(4,2)$ and $\beta=(1)$, then the disjoint union $\lambda / \alpha \sqcup \mu / \beta$ is

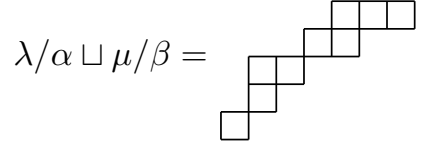

Let $\sigma$ be a connected skew diagram. For each $i \in \mathbb{Z}$, let $D_{i}(\sigma)=\{(a, b) \in \sigma \mid b-a=i\}$ be the $i$-th diagonal of $\sigma$. If $D_{i}(\sigma) \neq \varnothing$, let $x_{i}$ be the square in $D_{i}(\sigma)$ whose coordinate sum is bigger than the coordinate sums of all other squares in $D_{i}(\sigma)$. Since $\sigma$ is connected, the set $b(\sigma)$ of all such $x_{i}$ 's is a border strip. We call $b(\sigma)$ the principal border strip of $\sigma$.

Diagram classes 5.3 If $\sigma$ is a skew diagram, its diagram class $[\sigma]$ is the set of all skew diagrams isomorphic to $\sigma$. Let $D=[\lambda / \alpha]$. The size of $D$ is $|D|=|\lambda / \alpha|$ and the conjugate $D^{\prime}$ of $D$ is the diagram class $\left[\lambda^{\prime} / \alpha^{\prime}\right]$. If $E=[\mu / \beta]$ is another diagram class, denote $D \sqcup E=[\lambda / \alpha \sqcup \mu / \beta]$. Note that $D \sqcup E=E \sqcup D$. We say that $D$ is connected if $\lambda / \alpha$ is connected. If $D=[\sigma]$ is connected, then $B(D)=[b(\sigma)]$ is the principal border strip of $D$.

Removable diagrams 5.4 Let $\lambda$ be a partition. A skew diagram $\rho$ is $\lambda$-removable if there is a partition $\alpha \subseteq \lambda$ such that $\rho=\lambda / \alpha$. Define the set of $\lambda$-removable diagrams in a diagram class $D$ by

$$
\mathrm{R}_{\lambda}(D)=\{\rho \subseteq \lambda \mid \rho \text { is } \lambda \text {-removable and }[\rho]=D\},
$$

and let $r_{\lambda}(D)=\# \mathrm{R}_{\lambda}(D)$, be the number of elements in $\mathrm{R}_{\lambda}(D)$. For example, $\mathrm{r}_{\lambda}(\varnothing)=1$ and $\mathrm{r}_{\lambda}(\square)$ is the number of removable squares of $\lambda$ (see [5, p. 202]). Removable squares are also called corners of the diagram (see, for example, [24, p. 16]).

Conjugation of skew diagrams defines a bijection between the sets $\mathrm{R}_{\lambda}\left(D^{\prime}\right)$ and $\mathrm{R}_{\lambda^{\prime}}(D)$, therefore

$$
r_{\lambda}\left(D^{\prime}\right)=r_{\lambda^{\prime}}(D) \text {. }
$$

Example 5.5 Let $\lambda=(4,3,1)$, then $r_{\lambda}(\square)=3, r_{\lambda}(\square \sqcup \square)=2, r_{\lambda}(\bigoplus)=1$ and $r_{\lambda}(\square \sqcup \boxminus)=0$.

Collages of skew diagrams 5.6 Let $\lambda$ be a partition and $\sigma$ be a $\lambda$-removable diagram. A collage of $\sigma$ is a sequence $\left(\rho_{1}, \ldots, \rho_{m}\right)$ of $\lambda$-removable diagrams such that $\sigma=\cup_{i \in[m]} \rho_{i}$, where the union is not necessarily disjoint. For any finite list $D_{1}, \ldots, D_{m}$ of diagram classes define the set of collages of $\sigma$ determined by $D_{1}, \ldots, D_{m}$ by

$$
\mathrm{C}_{\lambda}\left(D_{1}, \ldots, D_{m} ; \sigma\right)=\left\{\left(\rho_{1}, \ldots, \rho_{m}\right) \in \mathrm{R}_{\lambda}\left(D_{1}\right) \times \cdots \times \mathrm{R}_{\lambda}\left(D_{m}\right) \mid \cup_{i \in[m]} \rho_{i}=\sigma\right\}
$$

and

$$
\mathrm{c}_{\lambda}\left(D_{1}, \ldots, D_{m} ; \sigma\right)=\# \mathrm{C}_{\lambda}\left(D_{1}, \ldots, D_{m} ; \sigma\right) .
$$

Let $\left(\rho_{1}, \ldots, \rho_{m}\right)$ be a collage of $\sigma$. Denote $D=[\sigma]$ and $D_{i}=\left[\rho_{i}\right]$. Then we say that $D$ is a collage of $D_{1}, \ldots, D_{m}$. In this case we have that $|D| \leq\left|D_{1}\right|+\cdots+\left|D_{m}\right|$. 
Lemma 5.7 Let $\alpha, \beta, \lambda, \mu$ be partitions such that $\alpha \subseteq \lambda$ and $\beta \subseteq \mu$. If $\lambda / \alpha$ and $\mu / \beta$ are isomorphic diagrams, then for any diagram classes $D_{1}, \ldots, D_{m}$ one has

$$
\mathrm{c}_{\lambda}\left(D_{1}, \ldots, D_{m} ; \lambda / \alpha\right)=\mathrm{c}_{\mu}\left(D_{1}, \ldots, D_{m} ; \mu / \beta\right) .
$$

Definition 5.8 Let $D_{1}, \ldots, D_{m}, D=[\lambda / \alpha]$ be diagram classes. Define

$$
\mathrm{c}\left(D_{1}, \ldots, D_{m} ; D\right)=\mathrm{c}_{\lambda}\left(D_{1}, \ldots, D_{m} ; \lambda / \alpha\right) .
$$

Because of Lemma 5.7 this definition does not depend on the representative of $D$. So, we can speak of the number of collages of $D$ determined by $D_{1}, \ldots, D_{m}$.

Proposition 5.9 Let $\lambda$ be a partition, $C_{1}, \ldots, C_{k}$ pairwise distinct connected diagram classes, $a_{1}, \ldots, a_{k}$ positive integers and $D=C_{1}^{\sqcup a_{1}} \sqcup \cdots \sqcup C_{k}^{\sqcup a_{k}}=D_{1} \sqcup \cdots \sqcup D_{m}$, where $m=a_{1}+\cdots+a_{k}$. Then

$$
\mathrm{r}_{\lambda}(D)=\frac{1}{a_{1} ! \cdots a_{k} !}\left[\mathrm{r}_{\lambda}\left(C_{1}\right)^{a_{1}} \cdots \mathrm{r}_{\lambda}\left(C_{k}\right)^{a_{k}}-\sum_{E,|E|<|D|} \mathrm{c}\left(D_{1}, \ldots, D_{m} ; E\right) \mathrm{r}_{\lambda}(E)\right] .
$$

Theorem 5.10 For any diagram class $D$ there is a polynomial $p_{D}\left(x_{C}\right)$ with rational coefficients, in the variables $x_{C}$, where $C$ runs over the set of connected diagram classes of size $|C| \leq|D|$, such that for all partitions $\lambda$ the number $r_{\lambda}(D)$ is obtained from $p_{D}\left(x_{C}\right)$ evaluating each $x_{C}$ at $\mathrm{r}_{\lambda}(C)$. So, we have

$$
r_{\lambda}(D)=p_{D}\left(r_{\lambda}(C)\right)
$$

If $D$ is not connected, the polynomial $p_{D}\left(x_{C}\right)$ depends only on the variables $x_{C}$ with $|C|<|D|$.

\section{The numbers $\operatorname{Ir}(\lambda, \lambda ; \pi)$}

Let $D=[\sigma]$ and $\pi \vDash|D|$. Then we set $\operatorname{lr}(D, D ; \pi)=\operatorname{Ir}(\sigma, \sigma ; \pi)$. This definition do not depend on the representative of $D$. For example, if $\Delta_{d}=\square \sqcup \cdots \sqcup \square$ is the disjoint union of $d$ squares, we have

Lemma 6.1 Let $\pi=\left(\pi_{1}, \ldots, \pi_{r}\right)$ be a composition of $d$. Then

$$
\operatorname{lr}\left(\Delta_{d}, \Delta_{d} ; \pi\right)=\left(\begin{array}{l}
d \\
\pi
\end{array}\right) d !=\frac{(d !)^{2}}{\pi_{1} ! \cdots \pi_{r} !} .
$$

We denote by $\mathcal{D}(d)$ the set of all diagram classes of size $d$.

Proposition 6.2 Let $\lambda$ be a partition of $n, \pi$ be a composition of $n$ and $d=|\bar{\pi}|$. Then

$$
\operatorname{Ir}(\lambda, \lambda ; \pi)=\sum_{D \in \mathcal{D}(d)} \operatorname{Ir}(D, D ; \bar{\pi}) \mathrm{r}_{\lambda}(D)
$$

The following result is a consequence of Proposition 6.2 and Theorem 5.10

Theorem 6.3 Let $\pi$ be a composition of $n$. Then, there exists a polynomial $q_{\bar{\pi}}\left(x_{C}\right)$ with rational coefficients in the variables $x_{C}$, where $C$ runs over the set of connected diagram classes of size $|C| \leq|\bar{\pi}|$, such that, for all partitions $\lambda$ of $n$, the number $\operatorname{Ir}(\lambda, \lambda ; \pi)$ is obtained from $q_{\bar{\pi}}\left(x_{C}\right)$ by evaluating each $x_{C}$ at $\mathrm{r}_{\lambda}(C)$. That is,

$$
\operatorname{lr}(\lambda, \lambda ; \pi)=q_{\bar{\pi}}\left(\mathrm{r}_{\lambda}(C)\right)
$$




\section{The Kronecker coefficients $\mathrm{g}(\lambda, \lambda, \nu)$}

The first result is an improvement of the RT method described in the introduction, that gives a closed combinatorial (up to signs) formula of Kronecker coefficients. It incorporates the notion of $\lambda$-removable diagram and a convenient use of special border strip tableaux. We will show its utility in Sections 8 and 9 .

Theorem 7.1 Let $\bar{\nu}$ be a partition of $d$ and $n \geq d+\nu_{2}$. Then, for any partition $\lambda$ of $n$, we have

$$
\mathrm{g}(\lambda, \lambda,(n-d, \bar{\nu}))=\sum_{k=0}^{d} \sum_{D \in \mathcal{D}(k)} \sum_{\substack{T \in \operatorname{SBST}(\widetilde{\nu}) \\ e(T)=|D|}} \operatorname{sgn}(T) \operatorname{lr}(D, D ; \bar{\tau}(T)) r_{\lambda}(D) .
$$

The next result is a consequence of Theorems 5.10 and 7.1

Theorem 7.2 Let $\bar{\nu}$ be a partition of $d$. Then there exists a polynomial with rational coefficients $k_{\bar{\nu}}\left(x_{C}\right)$ in the variables $x_{C}$, where $C$ runs over the set of connected diagram classes of size $|C| \leq d$, such that, for all $n \geq d+\nu_{2}$ and all partitions $\lambda$ of $n$, the Kronecker coefficient $\mathrm{g}(\lambda, \lambda,(n-d, \bar{\nu}))$ is obtained from $k_{\bar{\nu}}\left(x_{C}\right)$ by evaluating each $x_{C}$ at $\mathrm{r}_{\lambda}(C)$, that is,

$$
\mathrm{g}(\lambda, \lambda,(n-d, \bar{\nu}))=k_{\bar{\nu}}\left(\mathrm{r}_{\lambda}(C)\right) .
$$

Theorem 7.2 can be restated in the following way

Theorem 7.3 Let $\bar{\nu}$ be a partition of $d$. Then there exists a polynomial with rational coefficients $\widetilde{k}_{\bar{\nu}}\left(t_{B}\right)$ in the variables $t_{B}$, where $B$ runs over the set of border strip classes of size $|B| \leq d$, such that, for all $n \geq d+\nu_{2}$ and all partitions $\lambda$ of $n$, the Kronecker coefficient $\mathrm{g}(\lambda, \lambda,(n-d, \bar{\nu}))$ is obtained from $\widetilde{k}_{\bar{\nu}}\left(t_{B}\right)$ by evaluating each $t_{B}$ at $r_{\lambda}(B)$, that is,

$$
\mathrm{g}(\lambda, \lambda,(n-d, \bar{\nu}))=\widetilde{k}_{\bar{\nu}}\left(\mathrm{r}_{\lambda}(B)\right) .
$$

The following lemma appears in [38, p. 23]. It was rediscovered in [26]. Corollary 2.1 in [19] is a particular case of the lemma and can be derived from it.

Lemma 7.4 Let $n$, $d$ be such that $n \geq 2 d$. If $\lambda=\left(a^{b}\right)$ is a partition of $n$, then

$$
\mathrm{g}(\lambda, \lambda,(n-d, d))=\#\{\alpha \vdash d \mid \alpha \subseteq \lambda\}-\#\{\beta \vdash d-1 \mid \beta \subseteq \lambda\} .
$$

Proposition 7.5 Let $n, d \in \mathbb{N}$ be such that $n>d$. Then for any partition $\lambda$ of $n$ one has

$$
\mathrm{g}\left(\lambda, \lambda,\left(n-d, 1^{d}\right)\right)=\sum_{k=0}^{d}(-1)^{d-k} \sum_{D \in \mathcal{D}(k)}\left[\sum_{\alpha \vdash k} c_{\alpha}^{D} c_{\alpha^{\prime}}^{D}\right] \mathrm{r}_{\lambda}(D) .
$$

We recover with our techniques the following result that appears in [25, § 6]. Pak and Panova used this and Lemma 7.4 to prove some results on unimodality. From next lemma we can also obtain a more general version of Corollary 2.2 in [19]. 
Lemma 7.6 Let $d, n \in \mathbb{N}$ be such that $n>d$. If $\lambda=\left(a^{b}\right)$ is a rectangle partition of $n$, then

$$
\mathrm{g}\left(\lambda, \lambda,\left(n-d, 1^{d}\right)\right)=\sum_{k=0}^{d}(-1)^{d-k} \#\left\{\alpha \vdash k \mid \alpha \subseteq \lambda \text { and } \alpha=\alpha^{\prime}\right\} .
$$

Example 7.7 The polynomials $k_{\bar{\nu}}$ for $|\bar{\nu}| \leq 3$ are

1. $\mathrm{g}(\lambda, \lambda,(n))=1$.

2. $\mathrm{g}(\lambda, \lambda,(n-1,1))=\mathrm{r}_{\lambda}(\square)-1$.

3. $\mathrm{g}(\lambda, \lambda,(n-2,2))=\mathrm{r}_{\lambda}(\square)+\mathrm{r}_{\lambda}(\boxminus)+2\left(\begin{array}{c}\mathrm{r}_{\lambda}(\square) \\ 2\end{array}\right)-\mathrm{r}_{\lambda}(\square)$.

4. $\mathrm{g}\left(\lambda, \lambda,\left(n-2,1^{2}\right)\right)=\left[\mathrm{r}_{\lambda}(\square)-1\right]^{2}=2\left(\begin{array}{c}\mathrm{r}_{\lambda}(\square) \\ 2\end{array}\right)-\mathrm{r}_{\lambda}(\square)+1$.

5. $\mathrm{g}(\lambda, \lambda,(n-3,3))=\mathrm{r}_{\lambda}(\square \square)+\mathrm{r}_{\lambda}(\boxminus)+\mathrm{r}_{\lambda}(\square)+\mathrm{r}_{\lambda}(\square)$

$$
+\left[2 r_{\lambda}(\square)-3\right]\left[r_{\lambda}(\square)+r_{\lambda}(\boxminus)\right]+6\left(\begin{array}{c}
r_{\lambda}(\square) \\
3
\end{array}\right)-2\left(\begin{array}{c}
r_{\lambda}(\square) \\
2
\end{array}\right) .
$$

6. $\mathrm{g}(\lambda, \lambda,(n-3,2,1))=\mathrm{r}_{\lambda}(\boxminus)+\mathrm{r}_{\lambda}(\square)+\left[3 \mathrm{r}_{\lambda}(\square)-4\right]\left[\mathrm{r}_{\lambda}(\square)+\mathrm{r}_{\lambda}(\boxminus)\right]$

$$
+12\left(\begin{array}{c}
r_{\lambda}(\square) \\
3
\end{array}\right)-4\left(\begin{array}{c}
r_{\lambda}(\square) \\
2
\end{array}\right)+r_{\lambda}(\square) \text {. }
$$

7. $\mathrm{g}\left(\lambda, \lambda,\left(n-3,1^{3}\right)\right)=\mathrm{r}_{\lambda}(\boxminus)+\mathrm{r}_{\lambda}(\square)+\left[\mathrm{r}_{\lambda}(\square)-1\right]\left[\mathrm{r}_{\lambda}(\square)+\mathrm{r}_{\lambda}(\boxminus)\right]$

$$
+6\left(\begin{array}{c}
r_{\lambda}(\square) \\
3
\end{array}\right)-2\left(\begin{array}{c}
r_{\lambda}(\square) \\
2
\end{array}\right)+r_{\lambda}(\square)-1 \text {. }
$$

\section{Saxl conjecture}

In this section we present what we believe to be a surprising application of our enhancement of the RT method. Let $\rho_{k}=(k, k-1, \ldots, 2,1)$ be the staircase partition of size $n_{k}=\left(\begin{array}{c}k+1 \\ 2\end{array}\right)$. The Saxl conjecture states that for all $k \geq 1$ the Kronecker square $\chi^{\rho_{k}} \otimes \chi^{\rho_{k}}$ contains all irreducible characters as components. See [27] for more information about the conjecture and some results towards its proof. For each $k \geq 1$, let $\zeta_{k}=b\left(\rho_{k}\right)$ denote the principal border strip of $\rho_{k}$. Then $\left|\zeta_{k}\right|=2 k-1$. Denote also $Z_{k}=\left[\zeta_{k}\right]$.

Let $D$ be a diagram class and let $D=C_{1}^{\sqcup a_{1}} \sqcup \cdots \sqcup C_{m}^{\sqcup a_{m}}$ be a decomposition of $D$ into its connected components, such that $C_{1}, \ldots, C_{m}$ are pairwise distinct connected diagram classes and $a_{1}, \ldots, a_{m} \in \mathbb{N}$. We call such decomposition of $D$ a sorted decomposition.

For a partition $\bar{\nu}=\left(\nu_{2}, \ldots, \nu_{r}\right)$ of $d$ let $t(\bar{\nu})$ be the smallest integer that is greater or equal to $\frac{-1+\sqrt{1+8\left(\nu_{2}+d\right)}}{2}$. Hence $n_{k} \geq d+\nu_{2}$ if and only if $k \geq t(\bar{\nu})$. In other words, $\left(n_{k}-d, \bar{\nu}\right)$ is a partition if and only if $k \geq t(\bar{\nu})$. Finally, let $c(\bar{\nu})=\max \{d-1, t(\bar{\nu})\}$. 
Lemma 8.1 Let $D=C_{1}^{\sqcup a_{1}} \sqcup \cdots \sqcup C_{m}^{\sqcup a_{m}}$ be a sorted decomposition. Suppose that for each $i \in[m]$ there is some $n_{i}$ such that $B\left(C_{i}\right)=Z_{n_{i}}$. Let $k \in \mathbb{N}$ and denote $a_{0}=k-\sum_{i \in[m]} n_{i} a_{i}$. If $a_{0}<0$, then $\mathrm{r}_{\rho_{k}}(D)=0$. If $a_{0} \geq 0$, then

$$
\mathrm{r}_{\rho_{k}}(D)=\left(\begin{array}{c}
a_{0}+a_{1}+\cdots+a_{m} \\
a_{0}, a_{1}, \ldots, a_{m}
\end{array}\right) .
$$

Theorem 8.2 Let $\bar{\nu} \vdash d$. Then there is a piecewise polynomial function with rational coefficients

$$
s_{\bar{\nu}}:[0, \infty) \longrightarrow \mathbb{R}
$$

such that for all $k \geq t(\bar{\nu})$

$$
\mathrm{g}\left(\rho_{k}, \rho_{k},\left(n_{k}-d, \bar{\nu}\right)\right)=s_{\bar{\nu}}(k) .
$$

Moreover, $s_{\bar{\nu}}$ is a polynomial function of degree $d$ in the interval $[c(\bar{\nu}), \infty)$ with leading coefficient $f^{\bar{\nu}}$.

Lemma 8.3 Let $\bar{\nu}=\left(m, 1^{d-m}\right)$ be a partition of $d$. Then the coefficient of $x^{d-1}$ in $s_{\bar{\nu}}(x)$ in the interval $[c(\bar{\nu}), \infty)$ is $-f^{\bar{\nu}}\left[\left(\begin{array}{l}d \\ 2\end{array}\right)+1\right]$.

Theorem 8.4 Let $\bar{\nu}$ be a partition of $d$. Then $\mathrm{g}\left(\rho_{k}, \rho_{k},\left(n_{k}-d, \bar{\nu}\right)\right)>0$, for all $k \geq t(\bar{\nu})$, with the possible exception of at most $2 d-\frac{1+\sqrt{1+8\left(\nu_{2}+d\right)}}{2} k$ 's.

\begin{tabular}{|r||c|c|c|}
\hline $\bar{\nu}$ & $s_{\bar{\nu}}$ in the interval $[c(\bar{\nu}), \infty)$ & real roots of $s_{\bar{\nu}}$ & $t(\bar{\nu})$ \\
\hline \hline$(1)$ & $x-1$ & 1 & 2 \\
\hline$(2)$ & $x^{2}-2 x$ & 0,2 & 3 \\
$\left(1^{2}\right)$ & $x^{2}-2 x+1$ & 1,1 & 2 \\
\hline$(3)$ & $x^{3}-4 x^{2}+4 x-1$ & $0.38,1,2.62$ & 3 \\
$(2,1)$ & $x^{3}-8 x^{2}+8 x-1$ & $0.15,1.4,2.45$ & 3 \\
$\left(1^{3}\right)$ & $x^{3}-4 x^{2}+5 x-2$ & $1,1,2$ & 3 \\
\hline$(4)$ & $x^{4}-7 x^{3}+17 x^{2}-18 x+7$ & $1,3.32$ & 4 \\
$(3,1)$ & $3 x^{4}-21 x^{3}+51 x^{2}-51 x+18$ & $1,1,2,3$ & 4 \\
$\left(2^{2}\right)$ & $2 x^{4}-14 x^{3}+34 x^{2}-33 x+11$ & $0.81,1$ & 3 \\
$\left(2,1^{2}\right)$ & $3 x^{4}-21 x^{3}+52 x^{2}-53 x+18$ & $0.69,1.63,2,2.68$ & 3 \\
$\left(1^{4}\right)$ & $x^{4}-7 x^{3}+18 x^{2}-20 x+8$ & $1,2,2,2$ & 3 \\
\hline$(5)$ & $x^{5}-11 x^{4}+48 x^{3}-106 x^{2}+119 x-54$ & $1.56,2,3.79$ & 4 \\
$(4,1)$ & $4 x^{5}-44 x^{4}+192 x^{3}-420 x^{2}+462 x-203$ & $1.41,2.3,3.52$ & 4 \\
$(3,2)$ & $5 x^{5}-55 x^{4}+240 x^{3}-522 x^{2}+567 x-245$ & $1.42,2.46,3.27$ & 4 \\
$\left(3,1^{2}\right)$ & $6 x^{5}-66 x^{4}+289 x^{3}-632 x^{2}+690 x-300$ & $1.54,2,3.25$ & 4 \\
$\left(2^{2}, 1\right)$ & $5 x^{5}-55 x^{4}+241 x^{3}-526 x^{2}+571 x-246$ & $1.53,2,3$ & 4 \\
$\left(2,1^{3}\right)$ & $4 x^{5}-44 x^{4}+194 x^{3}-428 x^{2}-470 x-204$ & $1.41,2,3$ & 4 \\
$\left(1^{5}\right)$ & $x^{5}-11 x^{4}+49 x^{3}-110 x^{2}+124 x-56$ & $2,2,2$ & 3 \\
\hline
\end{tabular}

Tab. 1: First polynomial functions

From the information in Table 1 we conclude 
Corollary 8.5 Let $d \in[5]$ and $\bar{\nu} \vdash d$. Then for all $k \in[t(\bar{\nu}), \infty)$ the character $\chi^{\left(n_{k}-d, \bar{\nu}\right)}$ is a component of $\chi^{\rho_{k}} \otimes \chi^{\rho_{k}}$.

We propose two conjectures concerning the maps $s_{\bar{\nu}}$. The first implies Saxl conjecture.

Conjecture 8.6 Let $\bar{\nu} \vdash d$. Then $s_{\bar{\nu}}$ is positive in the interval $[t(\bar{\nu}), \infty)$.

Conjecture 8.7 Let $\bar{\nu} \vdash d$. Then the polynomial map $s_{\bar{\nu}}$ has nonzero integer coefficients in the interval $[c(\bar{\nu}), \infty)$ and its signs alternate. The sign of the coefficient of $x^{k}$ in $s_{\bar{\nu}}(x)$ is $(-1)^{d-k}$ for all $0 \leq k \leq d$.

\section{A stability property for Kronecker squares}

In this section we prove a new property for Kronecker coefficients. It generalizes, in the case of Kronecker squares, the stability property noted by Murnaghan [22] and proved since in different ways [7, 17, 35, 37]. In particular, our graphical approach yields a new proof of Murnaghan's stability property. It might be possible to find a proof of this new property using other techniques, but it was the graphical method exposed here that permitted its discovery.

Notation 9.1 For any partition $\mu=\left(\mu_{1}, \ldots, \mu_{q}\right)$, given $k \in \mathbb{N}$ and $i \in[q]$, denote

$$
\mu^{(i, k)}=\left(\mu_{1}+k, \ldots, \mu_{i}+k, \mu_{i+1}, \ldots, \mu_{q}\right) .
$$

Theorem 9.2 Let $\lambda, \nu$ be partitions of $n, k \in \mathbb{N}$ and $i \in[\ell(\lambda)]$. If $\lambda_{i}-\lambda_{i+1} \geq \mathrm{d}(\nu)$, then

$$
\mathrm{g}\left(\lambda^{(i, k)}, \lambda^{(i, k)}, \nu^{(1, k i)}\right)=\mathrm{g}(\lambda, \lambda, \nu) .
$$

\section{Acknowledgements}

I am grateful to Christine Bessenrodt, Igor Pak and Miguel Raggi for helpful suggestions. The package Sage [34] proved very useful in some of the computations done for the elaboration of Table 1 ]

\section{References}

[1] D. Avella-Alaminos and E. Vallejo, Kronecker products and the RSK correspondence, Discrete Math. 312 (2012), $1476-1486$.

[2] C. M. Ballantine and R. C. Orellana, On the Kronecker product $s_{(n-p, p)} * s_{\lambda}$, The Elec. J. of Comb. 12 (2005), \#R28 pp. 26.

[3] C. M. Ballantine and R. C. Orellana, A combinatorial interpretation for the coefficients in the Kronecker product $s(n-p, p) * s_{\lambda}$, Sém. Lothar. Combin. 54A (2006), Art. B54Af, 29 pp.

[4] Ch. Bessenrodt, On mixed products of complex characters of the double covers of the symmetric groups, Pacif. J. Math. 199 (2001), 257-268.

[5] Ch. Bessenrodt and A. Kleshchev, On Kronecker products of complex representations of the symmetric and alternating groups, Pacific J. Math. 190 (1999), 201-223.

[6] Ch. Bessenrodt and S. van Willigenburg, On (almost) extreme components in Kronecker products of characters of the symmetric groups. arXiv:1105.3170v2 [math.CO], 2013.

[7] M. Brion, Stable properties of plethysm: on two conjectures of Foulkes, manuscripta math. 80 (1993), 347-371.

[8] P. Bürgisser, J. Landsberg, L. Manivel and J. Weyman, An overview of mathematical issues arising in the geometric complexity theory approach to VP $\neq$ VNP, SIAM J. Comput. 40 (2011), 1179-1209.

[9] M. Christandl, G. Mitchison, The spectra of quantum states and the Kronecker coefficients of the symmetric group, Comm. Math. Phys. 261 (2006) 789-797. 
[10] I. F. Donin, Decompositions of tensor products of representations of a symmetric group and of symmetric and exterior powers of the adjoint representation of $\mathfrak{g l}(N)$. Soviet Math. Dokl. 38 (1989), 654-658.

[11] Ö. Eg̃eciog̃lu and J.B. Remmel, A combinatorial interpretation of the inverse Kostka matrix, Linear and Multilinear Algebra 26 (1990), $59-84$.

[12] A. Gamba and L.A. Radicati, Sopra un teorema per la riduzione di talune rappresentazioni del gruppo simmetrico, Atti Accad. Naz. Lincei. Rend. Cl. Sci. Fis. Mat. Nat. 14, (1953), 632-634.

[13] A.M. Garsia and J. Remmel, Shuffles of permutations and the Kronecker product, Graphs and Combin. 1 (1985), $217-263$.

[14] G.D. James and A. Kerber, "The representation theory of the symmetric group", Encyclopedia of mathematics and its applications, Vol. 16, Addison-Wesley, Reading, Massachusetts, 1981

[15] A. Klyachko, Quantum marginal problem and representations of the symmetric group, arXiv:quant-ph/0409113v1, 2004.

[16] D.E. Littlewood, The Kronecker product of symmetric group representations, J. London Math. Soc. 31 (1956), 89-93.

[17] D.E. Littlewood, Products and plethysms of characters with orthogonal, symplectic and symmetric groups, Canad. J. Math. 10 (1958), 17-32.

[18] I.G. Macdonald, "Symmetric functions and Hall polynomials", 2nd. edition Oxford Mathematical Monographs Oxford Univ. Press 1995.

[19] L. Manivel, On rectangular Kronecker coefficients, J. Algebr: Comb. 33 (2011), 153-162.

[20] K. D. Mulmuley and M. Sohoni, Geometric complexity theory. I, An approach to the P vs. NP and related problems, SIAM J. Comput. 31 (2001) 496-526.

[21] K. D. Mulmuley and M. Sohoni, Geometric complexity theory. II, Towards explicit obstructions for embeddings among class varieties, SIAM J. Comput. 38 (2008) 1175-1206.

[22] F.D. Murnaghan, The analysis of the Kronecker product of irreducible representations of the symmetric group, Amer. J. Math. 60 (1938), 761-784.

[23] F.D. Murnaghan, On the Kronecker product of irreducible representations of the symmetric group, Proc. Nat. Acad. Sci. USA 42 (1956), 95-98.

[24] I. Pak, Partition bijections, a survey, Ramanujan J. 12 (2006), 5-75.

[25] I. Pak and G. Panova, Unimodality via Kronecker products, arXiv:1304.5044, 2013.

[26] I. Pak and G. Panova, Strict unimodality of $q$-binomial coefficients, C. R. Acad. Sci. Paris, Ser. I 351 (2013), $415-418$.

[27] I. Pak, G. Panova and E. Vallejo, Kronecker products, characters, partitions, and the tensor square conjectures, arXiv:1304.0738 [math.CO], 2013.

[28] J.B. Remmel, A formula for the Kronecker products of Schur functions of hook shapes, J. Alg. 120 (1989), 100-118.

[29] G. de B. Robinson, "Representation theory of the symmetric group”. University of Toronto Press, 1961.

[30] G. de B. Robinson and O.E. Taulbee, The reduction of the inner product of two irreducible representations of $\mathrm{S}_{n}$, Proc. Nat. Acad. Sci. U.S.A. 40 (1954), 723-726.

[31] B. Sagan, "The symmetric group. Representations, combinatorial algorithms and symmetric functions". Second ed. Graduate Texts in Mathematics, 203. Springer Verlag, 2001.

[32] J. Saxl, The complex characters of the symmetric groups that remain irreducible on subgroups, J. Algebra 111 (1987), $210-219$.

[33] R. P. Stanley, "Enumerative Combinatorics, Vol. 2", Cambridge Studies in Advanced Mathematics 62. Cambridge Univ. Press, 1999.

[34] W. A. Stein et al., Sage Mathematics Software (Version 4.8), The Sage Development Team, 2011, http: //www. sagemath. org.

[35] J. Y. Thibon, Hopf algebras of symmetric functions and tensor products of symmetric group representations, Internat. J. Algebra Comput. 1 (1991), 207-221.

[36] E. Vallejo, On the Kronecker product of the irreducible characters of the symmetric group, Pub. Prel. Inst. Mat. UNAM 526, March 1997.

[37] E. Vallejo, Stability of Kronecker product of irreducible characters of the symmetric group, Electron. J. Combin 6 (1999) Reseach Paper 39, 7 pp.

[38] E. Vallejo, Kronecker squares of complex $S_{n}$ characters and Littlewood-Richardson multitableaux, manuscript, 2007.

[39] E. Vallejo, A diagramatic approach to Kronecker squares, arXiv: 1310.8362, [math.CO], 2013.

[40] I. Zisser, The character covering numbers of the alternating groups, J. Algebra 153 (1992), 357-372. 